\title{
Hemichorea in tuberculous meningitis
}

\author{
Hemicoreia na meningite tuberculosa \\ Marcus Vinicius Pinto, Tiago Aguiar, Renata Nogueira, Ana Carolina Dias, Ana Lúcia Rosso
}

We report on a 14 y/o HIV-negative girl with diagnosis of miliary tuberculosis (meningitis and pulmonary disease) who presented with right hemichorea three weeks after initiation of prednisone, rifampin, isoniazide, pyrazinamide and ethambutol. Brain MRI showed ischemic lesions in left caudate and lenticulo-capsular area (Figure). Hemichorea is a rare neurological manifestation of tuberculosis ${ }^{1}$. Basal

Figure. Coronal T2-weighted image shows hyperintensity in the left head of the caudate and lenticular nucleus (A). Axial T1weighted image with gadolinium shows enhancement without mass effect in left lenticular nucleus, suggestive of subacute ischemic injury (B). Axial diffusion-weighted image (DWI) and axial apparent diffusion coefficient (ADC) map show restricted diffusion compatible with acute ischemic injury (C, D). Axial T1weighted image with gadolinium shows a lacunar infarct in left internal capsule consistent with chronic ischemic injury. These changes are compatible with ischemic lesions in different stages (E). ganglia infarcts caused by vasculitis probably decreased the GABAergic transmission in indirect basal ganglion pathway causing hemichorea. The major differential diagnoses in acquired hemichorea are Sydenham's chorea, nonketotic hyperglycemia and cerebral toxoplasmosis ${ }^{2}$. We emphasize the importance of brain MRI in the diagnostic evaluation of these patients.

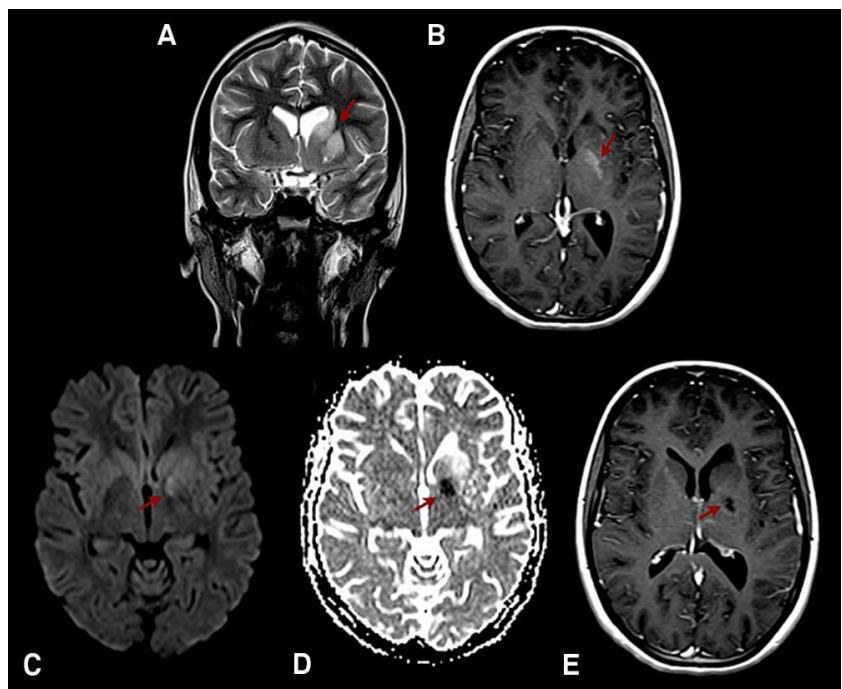

1. Alarcón F, Duenãs G, Cevallos N, Lees AJ. Movement disorders in 30 patients with tuberculous meningitis. Mov Disord 2000;15:561-569.
2. Wild EJ, Tabrizi SJ. The differential diagnosis of chorea. Pract Neurol 2007;7:360-373. 